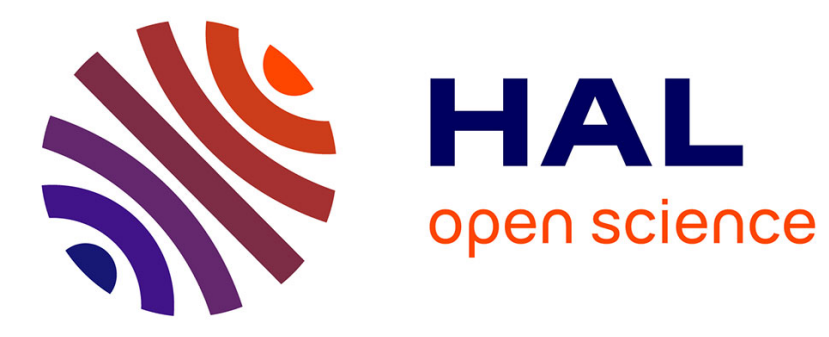

\title{
La Société de Linguistique de Paris (1870-1914)
}

Gabriel Bergounioux

\section{To cite this version:}

Gabriel Bergounioux. La Société de Linguistique de Paris (1870-1914). Bulletin de la Société de Linguistique de Paris, 1997, XCII (1), pp.1-26. halshs-01845144

\section{HAL Id: halshs-01845144 https://shs.hal.science/halshs-01845144}

Submitted on 20 Jul 2018

HAL is a multi-disciplinary open access archive for the deposit and dissemination of scientific research documents, whether they are published or not. The documents may come from teaching and research institutions in France or abroad, or from public or private research centers.
L'archive ouverte pluridisciplinaire HAL, est destinée au dépôt et à la diffusion de documents scientifiques de niveau recherche, publiés ou non, émanant des établissements d'enseignement et de recherche français ou étrangers, des laboratoires publics ou privés. 
G. Bergounioux Bulletin de la Société de Linguistique de Paris, t. XCII, 1, p. 1-26 (1997)

\section{LA SOCIÉTÉ DE LINGUISTIQUE DE PARIS (1876-1914)}

SOMMAIRE.- Après avoir trouvé son équilibre à partir de 1876 sous la direction de jeunes comparatistes spécialistes des langues indo-européennes, la Société de Linguistique de Paris devient le centre de référence des sciences du langage, accueillant des recherches qui sont peu représentées dans les facultés de lettres : la phonétique, la dialectologie et les argots, les langues celtiques, couchitiques ou "soudanaises». L'analyse des communications et des contributions parues dans les Mémoires de la société permet de marquer quel fut le thème dominant des discussions vers 1870 (la grammaire historique du français), 1880 (la métrique et l'étymologie latines), 1890 (la dialectologie et l'étymologie grecques) et dans les annces qui précédèrent la Première Guerre Mondiale (le comparatisme indo-iranien et le déchiffrement). Cette «anabase» dans le temps et dans l'espace (de l'ancien français aux représentants archaïques du groupe indo-iranien) s'accomplit sous l'influence des deux premiers secrétaires : Bréal (1832-1915) et Meillet (1866-1936).

En 1876, dix ans seulement après sa fondation, la Société de Linguistique de Paris (dorénavant : S.L.P.) apparaît comme le lieu par excellence de la recherche linguistique en France ${ }^{1}$. Alors que les facultés de lettres finalisent leurs formations pour se conformer aux nouveaux programmes de la licence, que le Collège de France et l'Ecole Pratique des Hautes Etudes (E.P.H.E.) ont des difficultés à retenir un public étudiant faute de délivrer un diplôme ou de garantir un déboùché professionnel, la S.L.P., affranchie des exi gences de collation des grades et des spécialisations obligées, se constitue comme le centre de référence d'une «école française de linguistique ».

\footnotetext{
${ }^{1}$ Bergounioux G., «Aux origines de la Société de Linguistique de Paris (1864-1876)», Bulletin de la Société de Linguistique de Paris, XCI, 1, 1996, p. 1-36.
} 
En effet, si la méthode génétique et le paradigme comparatiste indo-européen $\mathbf{2}$ orientent et unifient le champ de la linguistique au XIX ${ }^{\mathrm{e}}$ siècle, des différences entre écoles nationales s'accusent en même temps que se diffuse la grammaire historique en fonction des politiques linguistiques, des contacts coloniaux, du système universitaire, aussi selon la pondération relative entre disciplines. La phonétique (Rousselot), la psychologie (Sechehaye), la géographie linguistique (Gilliéron), la philosophie (V. Henry), la sémantique (Bréal), la logique (Couturat) contribuent au remodelage des sciences du langage $\mathbf{3}$ dont, à la veille de la Première Guerre mondiale, Vendryes résume les acquis, les enjeux et les impasses dans Le Langage, introduction linguistique à l'histoire4.

Le fonctionnement de la S.L.P. reflète à sa manière les contraintes inhérentes d'une association dont les adhérents sont surtout des ressortissants et qui ne peut compter, pour son recrutement, que sur un très petit nombre d'institutions: I'effectif forcément restreint des participants actifs appartenant au même cercle de professeurs parisiens implique une corrélation étroite entre l'organisation de l'enseignement supérieur et la distribution des responsabilités de direction. Dans l'effort accompli pour décrire de nouvelles langues, l'évolution des discussions semble moins se conformer à l'expansion coloniale (ou du moins avec un considérable retard) qu'à l'investissement scientifique accompli singulièrement par tel ou tel qui fait profiter la société de ses compétences.

L'activité de la S.L.P. peut être appréhendée comme une interaction entre deux relations :

- d'un point de vue institutionnel, le rapport, institué au travers de la délégation de pouvoir, entre les adhérents et les dirigeants qui ont en charge d'«incarner» la linguistique auprès des autorités ;

- d'un point de vue scientifique, la dévolution à un secteur restreint de l'enseignement supérieur de la décision d'orienter les thèmes de recherche tels qu'on les trouve consignés dans le Bulletin et les Mémoires.

L'interférence entre le contrôle associatif et la coincidence universitaire devient plus évidente encore dans la manière dont Meillet organise la S.L.P.

\section{LA VIE DE LA SOCIÉTÉ DE LINGUISTIQUE DE PARIS}




\subsection{Les dirigeants}

Dans le champ de la linguistique, une certaine concurrence semble perdurer entre plusieurs groupements. Néanmoins, la Société d'Ethnographie et la Société d'Anthropologie demeurent des associations d'amateurs et à ce titre sont disqualifiées par le caractère professionnel d'une S.L.P. où se retrouvent, après qu'elle a été investie par les comparatistes, des professeurs du supérieur. Devenue l'endroit privilégié pour la discussion des questions de langue, la S.L.P. se distingue encore d'autres associations proches de l'université comme la Société Asiatique et l'Association pour l'Encouragement des Etudes Grecques en France (A.E.É.G.F.) où il est traité autant et plus d'histoire et de civilisation que de grammaire, recrutant leurs adhérents plutôt à l'École des Langues Orientales pour la première, dans les lycées pour la seconde. La Société Asiatique réunit quelques savants prestigieux ayant chacun leur domaine propre (Cordier en sinologie, Maspero en égyptologie, James Darmesteter en indianisme ou Jules Oppert en études sémitiques), rivalisant avec leurs pairs en Europe et guère sollicités par leurs étudiants ; l'hétérogénéité des groupes linguistiques rassemblés dans la Société Asiatique l'empêche d'afficher une cohérence comparable à celle de la S.L.P. L'A.E.É.G.F. a le problème inverse: se déclarant en faveur d'une seule langue, elle est entravée dans ses velléités d'innovation philologique par l'importance numérique d'adhérents issus du second degré plus sensibles à la tradition d'excellence des humanités qu'à un " réductionnisme » indo-européen. Comme l'indique le tableau 1, Les dirigeants de la S.L.P. se recrutent principalement parmi les titulaires de chaires d'établissements d'enseignement supérieur — mais en dehors des facultés de lettres - dans lesquelles ils traitent de disciplines constituées au cours du XIX ${ }^{\mathrm{e}}$ siècle (le grec moderne et le sanscrit par exemple) ou traditionnelles (comme le grec ancien et l'hébreu) par la méthode comparatiste. Issus d'un réseau extrêmement restreint de postes et par une translation du marché universitaire, les responsables élus au bureau de la S.L.P.5 et au comité de publication appartiennent de préférence au Collège de France et à 1'É.P.H.É., ce qui exclut les enseignants de lettres, latinistes ou hellénistes, en poste à la Sorbonne ou dans les universités de province.

Un partage des tâches rigoureux s'effectue au bureau. Bréal, à titre de secrétaire, supervise les activités et joue de ses relations pour obtenir subventions, locaux et reconnaissance d'intérêt public, assumant le rôle assuré par Egger durant les premières années. A l'administrateur est dévolu le fonctionnement au quotidien (convocations, procès-verbaux des séances, publication du Bulletin...), au trésorier et au 
bibliothécaire des charges subalternes. Le président passe et ne gouverne pas ; sont élus à ce titre, à côté de savants prestigieux, des universitaires et des amateurs dont les travaux n'ont pas marqué l'histoire de la linguistique : Eugène Benoist (1877), Robert Mowat (1878), Hyacinthe de Charencey (1885), Charles Ploix (1889), Alexandre Bibesco (1894)...

Tableau $\mathrm{N} \cdot 1$ Appartenance institutionnelle des membres du bureau de la S.L.P.

$\begin{array}{lrrrr}\text { Année } & 1885 & 1895 & 1905 & 1907 \\ \begin{array}{l}\text { Fonction } \\ \text { Secrétaire }\end{array} & \text { Bréal } & \text { Bréal } & \text { Bréal } & \text { Bréal } \\ \text { Sre adjoint } & \text { Saussure } & & & \text { Meillet } \\ \text { Administrateur } & \text { Psichari } & \text { Duvau } & \text { Gauthiot } & \text { Gauthiot } \\ \text { Trésorier } & \text { Berger } & \text { Finot } & \text { Cart } & \text { Cart } \\ \text { Bibliothécaire } & \text { Baize } & \text { Cart } & \text { Chilot } & \text { Chilot }\end{array}$

En gras (Bréal): professeur au Collège de France. En italiques (Saussure): professeur à l'E.P.H.E (IV section). Bréal appartient aux deux institutions.

Source : Bulletin de la S.L.P. 
Le Comité de publication (tableau 2) a en charge l'édition des Mémoires qui constituent, en langue française, la première revue de référence en matière de linguistique, les contributions de second ordre étant reléguées dans le Bulletin. Sa composition se modèle sur la hiérarchie des langues valorisées par le comparatisme universitaire sans que la réartition soit formalisée par un réglement. Si le grec semble faire exception en étant absent de la liste, on peut penser que la présence de Bréal (le bureau est membre de droit du comité) y supplée. 
Autant les premières années suivant la fondation avaient été difficiles, autant le système de cooptation assure efficacement la pérennité de la S.L.P.: ceux qui en prennent la direction entre 1868 et 1872 sont jeunes - de trente-six ans (Bréal) à vingt-neuf ans (Gaston Paris) - et ceux de la génération précédente qui les ont aidés s'effacent bientôt devant eux. Dans la seule année 1885 disparaissent Frédéric Baudry, Émile Egger et Léon Renier sans qu'aucune crise ne survienne, la relève s'étant accomplie dix ans auparavant.

La représentativité et plus encore l'autorité institutionnelle de la direction garantissent sa longévité. Les seuls qui pourraient prétendre rivaliser avec les responsables de la S.L.P. sont obligatoirement leurs élèves, 
voués, un jour ou l'autre, à être notés lors d'une inspection, à dépendre d'eux pour une promotion ou un recrutement. Il aurait fallu quelque affrontement extra-scientifique pour qu'une crise divise les dirigeants et forme, à travers d'autres alliances, de nouvelles majorités mais la direction s'abstient de toute prise de position militante en dépit de l'engagement dreyfusard de plusieurs de ses membres.

TABLEAU N•2 Appartenance institutionnelle du comité de publication de la S.L.P. 


$\begin{array}{llclrrr}\begin{array}{l}\text { Domaine } \\ \text { Année }\end{array} & \text { Celtique } & \text { Sanscrit } & \text { Latin } & \text { Roman } & \text { Sémitique } & \text { Slavistique } \\ 1885 & & & & & & \\ 1895 & \text { d'Arbois } & \text { Bergaigne } & \text { Havet } & \text { G. Paris } & \text { Renan } & \\ 1905 & \text { d'Arbois } & \text { V. Henry } & \text { Havet } & \text { G. Paris } & \text { R. Duval } & \\ \text { d'Arbois } & \text { V. Henry } & \text { Havet } & \text { A. Thomas } & \text { R. Duval } & \text { L. Léger }\end{array}$


«d'Arbois» = d'Arbois de Jubainville. En gras (Renan) : professeur au Collège de France. En italiques (Bergaigne): professeur à l'E.P.H.E (IV ${ }^{\mathfrak{e}}$ section) (Havet et G. Paris appartiennent aux deux institutions).

Source: Bulletin de la S.L.P.

1.2. Les adhérents 
Le tableau 3 présente une situation d'ensemble des adhérents de la S.L.P. au terme de quarante années de fonctionnement, avant que la disparition de la première génération de comparatistes et la guerre ne changent les conditions d'exercice. Pour deux cent trente-six cotisants, on dénombre une trentaine d'institutions (étrangères pour plus de la moitié) et une soixantaine d'adhérents non ressortissants. La distribution des cent quarante adhérents français donnée à titre d'exemple indique une proportion de deux tiers d'enseignants (dont les deux tiers en poste dans l'enseignement supérieur). Les élections ayant lieu à Paris, le coût des déplacements et la disponibilité suffisent à expliquer l'efficacité des procèdures de cooptation. Seuls les professeurs de lycée auraient pu, s'ils s'étaient inscrits en grand nombre, former un 
groupe de pression dont la présence aurait compromis la domination du bureau mais la S.L.P. ne s'est jamais vraiment ouverte aux enseignants du second degré. Elle n'a réussi à s'implanter dans les lycées ni par le contenu des programmes, ni par le recrutement. Les commissions pédagogiques qui ont fait appel à tellement de ses membres éminents, où Bréal a plus souvent qu'à son tour donné l'impulsion, ne proposent plus d'application directe du comparatisme après l'échec essuyé dans l'enseignement de l'ancien français entre 1875 et 18856 . 
Dans le même temps, le recrutement de nouveaux adhérents est rendu difficile faute de postes de linguistique à proposer dans l'enseignement supérieur. Les comparatistes sont parvenus jeunes aux emplois les plus prestigieux. En 1880, Michel Bréal (Collège de France) a quarante-huit ans, Gaston Paris (É.P.H.É. et Collège de France) quarante et un ans, Paul Meyer (Chartes et Collège de France) quarante-deux ans et Arsène Darmesteter (Sorbonne) trente-quatre. Avec peu de créations, guère de mobilité et pas de retraite, les étudiants les plus brillants ont toutes les raisons de se détourner d'un cursus redouté pour ses exigences au profit d'un enseignement secondaire plus socialement prestigieux et plus rémunérateur. Que des personnalités tels Meillet, à Langues O., ou Gauthiot, longtemps professeur 
d'allemand en lycée, n'aient pu obtenir rapidement un poste digne de leur mérite dans l'enseignement supérieur traditionnel avait de quoi en décourager de moins bons, et ce fut le cas.

A partir de 1875, la croissance de l'enseignement supérieur est soutenue mais l'expansion concerne plutôt les facultés que les grands établissements. Les admissions à la S.L.P. s'en ressentent. Durant les années 1885-1895, alors que plusieurs dizaines d'amateurs et de polygraphes adhèrent, peu de linguistes connus sont admis : on relève les noms de l'arabisant René Basset (1888) et de trois phonéticiens, l'abbé Jean-Pierre Rousselot (1886), Maurice Grammont (1889) et Paul Passy (1892). L'absence de cours de 
linguistique à l'université est particulièrement sensible. En dehors de Paris, en 1914, la linguistique, la grammaire, la philologie et la dialectologie représentent moins de douze chaires et maîtrises de conférences en sorte que, paradoxalement, cinquante annces après sa fondation, la S.L.P. est retournée à une situation homologue à celle de ses débuts, rassemblant une minorité de linguistes face à une majorité de non spécialistes. Il y a cependant une différence d'importance : ceux-ci sont désormais des fonctionnaires et non plus des rentiers.

Tableau N•3 Origine déclarée des adhérents de la S.L.P. en 1911 
Total des adhérents

Institutions

dont institutions étrangères

Adhérents individuels

dont adhérents étrangers

Adbérents individuels étrangers 
dont 50 déclarent être enseignants.

11 Allemands

10 Suisses

6 Russes (et 1 Finlandais) et 6 Austro-Hongrois

5 Britanniques et 5 Belges 
3 Américains et 3 Néerlandais

2 Danois et 2 Italiens

1 Argentin, Cubain, Mexicain, Norvégien, Suédois.

Adhérents individuels français

20 ne déclarent pas d'activité précise,

10 exercent diverses professions en dehors de la fonction publique;

14 sont fonctionnaires mais non enseignants. 
99 appartiennent ou sont rattachés à 1 'Instruction Publique:

56 enseignent dans le supérieur (du professeur au répétiteur),

32 enseignent en lycée,

11 sont archivistes, conservateurs, administrateurs...

2. L'ACTIVITE SCIENTIFIQUE DE LA S.L.P. 
Pour comprendre diachroniquement l'évolution du recrutement et des centres d'intérêt de la S.L.P. de 1880 à la veille de la Première Guerre mondiale, on a répertorié dans un tableau les intervenants (Tableau 4), leur poids relatif dans l'association (Tableaux 5 et 6 ) et le contenu thématique de leurs interventions (Tableau 7) en exploitant dans deux diagrammes les données recueillies (Tableaux 8 et 9). Ces représentations ont été élaborées à partir du dépouillement exhaustif des comptes rendus de séances consignés dans le Bulletin de la S.L.P. durant cinq années (1880, 1890, 1900, 1910 et 1911). Le point de départ correspond au moment où la société, ayant dépassé la série des crises qui ont marqué sa création, accompagne le développement rapide de l'université. Passé 1878, le choix de l'année 1880 est assez 
indifférent (la prédilection pour le nombre rond suffit à l'expliquer) ; l'écart entre deux années-témoins, correspondant à une décennie, permet de rendre plus sensibles les changements qui apparaissent, que ce soit parmi les intervenants ou dans l'évolution des problématiques scientifiques majeures. Concernant la dernière période (1910-1911), la recension s'étend sur deux années afin d'intégrer un nombre comparable de séances puisque leur fréquence, de bimensuelle, est devenue mensuelle.

L'unité comptabilisée dans les tableaux 4 à 8 est la "communication », c'est-à-dire la présentation, généralement brève (une dizaine de minutes à trois quarts d'heure), d'une question linguistique préparée à 
l'avance par un sociétaire, suivie ou non d'une discussion. Ceci exclut les discours de célébration, les rapports administratifs ou financiers et les présentations succinctes d'ouvrages offerts à la société, l'échange scientifique prévalant sur l'échange social. Bien que le hasard des biographies, la conjoncture scientifique ou le renouvellement des adhérents aient pu modifier la pondération dans l'intervalle d'une décennie, que d'autres transformations aient pu advenir qui n'apparaîtront pas, les tendances lourdes de l'histoire de la S.L.P. gagnent, par ce moyen, en lisibilité.

2.1. Les producteurs (tableaux 4 à 6) 
Afin de mieux distinguer les producteurs majeurs, on a consigné le nom de tous ceux qui avaient produit au moins trois communications au cours des cinq années témoins. Ce seuil de trois interventions permet d'éliminer des linguistes relativement isolés dont l'exposé aurait dû, pour des raisons matérielles, se dérouler

sur deux séances consécutives. Bien qu'ils ne figurent pas nominativement, les autres contributeurs sont décomptés sous la rubrique «Autres intervenants». Pour les communications, on a porté sur une ligne le total de celles présentées par les linguistes nommés et, sur la ligne suivante, le total de celles présentées 
par des adhérents j qui sont intervenus à moins de trois reprises. À la dernière ligne est précisée la moyenne (arrondie) du nombre d'interventions faites par chacun des producteurs non nommés. Pour 1880, les cinq

dernières lignes du tableau se comprennent ainsi7: vingt et une personnes ont présenté une communication, 9 en ont présenté trois ou plus et 12 seulement une ou deux. Les 9 principaux intervenants ont délivré 48 communications et les 12 autres 15, soit 1,2 en moyenne pour chacun de ceux-ci. 
Premier constat, les « autres intervenants », qui représentent plus de la moitié de l'effectif en 1880 et les deux tiers en 1910, comptent pour un tiers entre ces deux dates, comme si la S.L.P. avait eu quelques difficultés, après avoir découragé les amateurs présents à ses débuts, à intéresser de nouveaux adhérents à ses discussions. 
Les tableaux 5 et 6 montrent comment, à une phase initiale d'équilibre, succède une période de repli durant laquelle les producteurs majeurs accaparent le temps de communication, avant un renversement de la proportion entre «autres intervenants» et «intervenants majeurs » dont le cercle s'est progressivement resserré autour de Meillet. Les proportions dans les communications varient corrélativement: alors que les principaux intervenants représentent de $75 \%$ à $90 \%$ des communications jusqu'à 1900, à partir de 1910 , le partage entre les deux groupes se rapproche d'un partage 60/40.

La permanence d'un certain nombre de producteurs sur une période qui équivaut à celle d'une générationtrente ans-

Tableau $\mathrm{N}^{\circ} 4$ Les producteurs de la S.L.P.

$\begin{array}{llllll}\text { Année } & 1880 & 1890 & 1900 & 1910 / 1911 & \text { Total }\end{array}$

Producteurs

L. Havet

G. Paris

$\mathrm{Ph}$. Berger

Loth

Joret

d'Arbois de Jubainville

4

Rochemonteix

Schwob

Mohl

Bréal

Charencey

Halévy

Meillet

5

4

327

V. Henry

4

7

4

4

4

3

3

Delafosse

\section{9}

21

L. Duvau

Gauthiot 
Vendryes

I. Lévy

Homburger

Intervenants cités

Autres intervenants

Interventions citées

$\mathrm{Nb}$ moyen d'interventions

$\begin{array}{ccccc} & & 4 & 2 & 6 \\ & & & 3 & 3 \\ & & & 3 & 3 \\ 9 & 10 & 10 & 8 & 20 \\ 12 & 4 & 5 & 17 & 36^{*} \\ 48 & 40 & 39 & 28 & 155 \\ 1,25 & 1 & 1,4 & 1,1 & 1,2\end{array}$

* Un "autre intervenant" ayant pu parler deux fois (par exemple en 1880 et en 1890), on ne peut en faire la somme par la simple addition des colonnes précédentes. 


témoigne du rôle central joué par de jeunes linguistes dans la création de la S.L.P., comme un équivalent, dans des conditions sociales et scientifiques il est vrai très différentes, du mouvement des Junggrammatiker. Si les transformations imposces en Allemagne par les néo-grammairiens dans la théorie comparatiste et le pouvoir universitaire n'ont guère eu de répercussions directes en France, c'est pour autant qu'un mouvement comparable, à la fois scientifique et social, avait déjà donné au champ de la linguistique un équilibre fondé sur le partage des institutions (le Collège de France et 1'É.P.H.É aux comparatistes, les facultés de lettres aux philologues traditionnels), des associations et des savoirs.

La victoire remportée par ces universitaires précocement férus de comparatisme et par ceux qu'ils cooptent (Saussure, Meillet, Gauthiot) permet de distinguer quatre profils d'intervenants:

—les dirigeants: ils contrôlent institutionnellement la S.L.P. et décident de l'orientation des discussions, directement—c'est le cas de Bréal, Havet, Meillet et Gauthiot—ou par l'intermédiaire de leurs élèves;

—les spécialistes dont la collaboration est irrégulière; souvent assez importante en début de carrière, quand la S.L.P. représente pour eux une logitimation indispensable et un premier lieu d'accueil, elle se raréfie sitôt que le champ d'activité qui leur est propre a acquis une autonomie suffisante. C'est l'attitude d'un romaniste comme Gaston Paris, d'un latiniste comme L. Havet, d'un égyptologue comme Gaston Maspero;

—les « rivaux», dont les caractéristiques sont assez proches des dirigeants mais qui sont entrés en opposition avec eux, par exemple Philippe Berger, professeur à la faculté protestante de théologie, ou Charles Joret, à qui des tentatives malheureuses pour introduire précocement en France les théories néo-grammairiennes coûteront une carrière parisienne. I1 finira professeur de littérature étrangère à Aix-en-Provence;

- un hapax, le comte de Charencey, qui se prévaut de son titre de fondateur puis de sa qualité de vétéran pour prononcer imperturbablement des interventions sans valeur scientifique.

Chacun des contributeurs majeurs représente une variante particulière de l'un de ces modèles, élève des maîtres, défricheur de domaines nouveaux, concurrent malchanceux ou —mais de moins en moinsamateur déconsidéré. 


\subsection{Les domaines (tableaux 7 à 9)}

Avec la même définition statistique, le tableau 7 réunit les données concernant les productions. Deux présentations étaient possibles selon qu'on donnait la préférence à des disciplines - la grammaire, la lexicologie, l'étymologie...—ou à des familles linguistiques. La deuxième solution a paru plus consistante. $\mathrm{Au} \mathrm{x} \sim \mathrm{xe}$, la recherche en linguistique tire plutôt sa légitimité d'un travail centré sur la description diachronique d'une langue (ou d'un groupe de langues) particulière que de l'étude construite sur un niveau d'observation: les transformations des sons, des mots ou des phrases. L'approche historique ou comparée adapte à des unités plus ou moins longues (du phonème à la proposition) une technique homologue de rapprochements et de correspondances et la distribution en disciplines a peu à faire de la distinction des méthodes du phonéticien, du grammairien — elles se confondent dans la morphologie — et du sémanticien (c'est à peine si le mot existe). En somme, privilogier l'aspect thématique reviendrait à projeter rétrospectivement des oppositions encore mal assurées.

La portée heuristique du comparatisme se mesure d'ailleurs à sa capacité à périmer les agrégats géographiques traditionnels («lan 
gues africaines», «langues balkaniques» ou «langues orientales») au profit de regroupements génétiques qui ne laissent subsister les apparentements anciens que dans la dénomination d'institutions. Seule exception, la phonétique, constituée en marge de la linguistique à partir des traitements acoustiques ou physiologiques de la parole issus des laboratoires, relève d'un programme indépendant de la reconstruction. Depuis Bopp, la langue particulière étudiée par le linguiste le définit, jusque dans les attentes du champ scientifique: on ne requiert pas d'un sémitisant ce qu'on demande à un sinologue ni d'un sanscritiste la sorte de travail à quoi s'astreint le latiniste. Cette façon de classer provoque un certain gauchissement sta tistique dès lors qu'un exposé porte SUF deux ou plusieurs langues. Si l'une des langues est privilégiée, elle est créditée de la commu nication; dans le cas contraire (e.g. «le latin et les langues roma nes »), elle est portée au compte de la langue la plus étudice puisqu'elle s'inscrit dans un domaine d'intérêt majeur. Au demeu rant, le cas n'est pas si fréquent. Les langues ou les groupes lin guistiques dont il est traité devant la S.L.P. sont répertoriés dans les tableaux dès qu'ils sont mentionnés à trois reprises et pour autant que, sous une forme ou sous une autre, ils sont présents dans au moins un établissement d'enseignement supérieur. Le basque a cependant été ajouté et le français distingué des autres langues romanes.

Les «autres langues» sont:

—en 1880: langues amérindiennes;

-en 1890: langues d'Asie;

—en 1900: l'arménien;

—en 1910: les langues amérindiennes, le malgache, les langues caucasiques et le turc (2 interventions).

Dans chaque case, le premier chiffre indique le nombre total d'interventions ayant trait à une langue donnée; le second, entre parenthèses, mentionne le nombre des intervenants. Une division permet alors de déduire le nombre moyen de communications par intervenant. Par exemple, la première case du tableau 7 se lit de la façon suivante:

«En 1880, quatre communications concernant le français ont été 
prononcées par trois intervenants, soit 1,33 communication par inter venant. »

Deux remarques concernant ces chiffres. En valeur absolue, $\_$s1ir $\sim 1 \sim$. 
alors que le nombre d'adhérents individuels reste constant à 10\% près entre 1880 et 1914, stabilisé aux environs de deux cents membres, le nombre d'interventions varie et après un maximum de 63 en 1880 (55 en 1872), se stabilise à 44 en 1890, 46 en 1900 et 47 en 1910-1911. Deuxièmement, le commentaire doit tenir compte de l'exiguïté de l'échantillon de référence. L'activité d'un savant suffit à modifier les statistiques: en 1880, le latiniste Louis Havet et le sémitisant Philippe Berger ont prononcé un tiers des communications; en 1900, quoiqu'ils appartiennent toujours à la S.L.P. ils n'y interviennent plus. Ceci explique en partie que l'attention portée au latin et aux langues sémitiques chute de moitié en vingt ans. Le détail, à intervalle de dix ans, permet de récapituler l'évolution de l'orientation des intérêts scientifiques.

1880: les rivalités jumelles

A cette date, la S.L.P. se veut le centre de diffusion en langue française du comparatisme indo-européen (langues romanes et sanscrit mais surtout latin, grec et langues celtiques) et sémitique. Sept langues (ou groupes de langues) ont fait l'objet de plus de deux communications (sont énumérés à la suite les producteurs ayant parlé deux fois ou plus), soit pour un total de 63 communications:

-le français: 4 interventions (2 pour F. Godefroy);

—le latin: 18 interventions (11 pour L. Havet, 4 pour M. Bréal);

—le grec: 3 interventions;

—l'indo-européen et les langues indo-iraniennes: 5 interventions ( 2 pour J. Halévy);

—les langues chamito-sémitiques et couchitiques: 15 interventions ( 8 pour Ph. Berger, 5 pour J. Halévy, 2 pour G. Maspero);

-les langues celtiques: 10 interventions (4 pour J. Loth, 3 pour d'Arbois de Jubainville);

—les langues amérindiennes: 3 interventions ( 3 pour H. de Charencey).

Le latin, les langues celtiques et chamito-sémitiques qui totalisent $65 \%$ des communications et atteignent ou dépassent la dizaine d'interventions, doivent ce surcroît relatif d'activité à la concurrence de deux linguistes:

-en latin,

Louis Havet, spécialiste de métrique et de prosodie, est confronté à Michel Bréal qui présente les informations tirées de

${ }_{-} \sim \mathrm{m}: \_\sim 7 \sim \sim_{-} \cdot-$ 
la rédaction du Dictionnaire Étymologique du Latin entrepris sous sa direction à l'École des Hautes Études; tous deux s'accordent à réévaluer une langue dont la fonction de distinction scolaire entre primaire et secondaire, entre établissements masculins et féminins, a routinisé l'usage dans des exercices de thème et de version. En mettant l'accent sur le lexique (l'étymologie) et la phonétique (la métrique) du latin, ils tentent d'imposer une redéflnition de son enseignement conforme à celui qu'ils pratiquent à 1'É.P.H.É et au Collège de France, une approche moins littéraire à laquelle les facultés de lettres restent réticentes8.

—dans les langues celtiques,

la démarche d'Arbois de Jubainville, un chartiste parisien initié au comparatisme (sa formation inclut le latin et c'est un des premiers spécialistes français des langues germaniques), travaillant sur documents, s'oppose à celle de Joseph Loth, un Breton bretonnant, plus intéressé par l'état contemporain des langues celtiques et la dialectologie armoricaine, dans un domaine en cours d'institutionnalisation. Le premier reçoit une chaire au Collège de France en 1882, le second un cours à la faculté des lettres de Rennes en 1883.

—dans les langues sémitiques,

en l'absence de personnalités issues du haut enseignement catholique, Philippe Berger, un universitaire classique qui enseigne les langues bibliques au séminaire protestant, s'oppose à Joseph Halévy, un ressortissant ladino d'Asie Mineure, venu en France avec le soutien de l'Alliance Israélite Universelle, si l'objet est commun, la tradition exégétique réformée et la définition du groupe chamito-sémitique relèvent de deux paradigmes hétérogenes.

1890: arrivée de Meillet

Sur 44 interventions, les trois quarts concernent quatre domaines dont deux où se distingue une seule personne:

—le latin (et les langues italiques): 11 interventions (8 pour Bréal [Meillet, V. Henry et Dutilleul une chacun]);

—le français: 7 interventions (4 pour M. Schwob qui trouve à 
la S.L.P. un accueil pour une analyse de l'argot peu prisée des romanistes).

Deux autres secteurs sont l'occasion d'une certaine rivalité entre deux producteurs:

-les langues sémitiques: 7 interventions ( 3 pour Ph. Berger, 3 pour J. Halévy). Berger s'intéresse plutôt au domaine punique et à l'histoire de l'écriture, Halévy aux relations des langues sémitiques orientales avec les parlers indo-iraniens;

—le grec: 10 interventions (4 pour A. Meillet, 2 pour V. Henry).

Alors que Meillet propose une analyse fondée sur des rapprochements avec les langues orientales du groupe indo-européen (notamment l'arménien) qu'il est un des rares à maîtriser, V. Henry s'intéresse plutôt aux aspects dialectaux. On note qu'en une décennie, la part accordée au grec dans les séances de la société a rattrapé celle consacrée au latin et que l'étude de l'indo-européen a laissé la place à des analyses spéciales langue par langue.

Cette recension montre l'autonomie de la S.L.P. à l'égard de la politique linguistique de la Troisième République. Malgré le développement de l'empire colonial, les langues de l'Afrique Noire et de la péninsule indochinoise n'y font pas l'objet de discussion et, dans le temps où l'œuvre scolaire de Ferry accélère la francisation de la Bretagne, des Flandres et du Midi, les langues celtiques, romanes et germaniques comptent deux communications à elles trois. La linguistique a couquis une autonomie suffisante pour déterminer son propre programme de recherches.

1900: la ruée vers 1'Est

Pour 46 communications présentées, les discussions concernent, par ordre décroissant:

-les langues celtiques: 9 interventions (4 pour Vendryes, 3 pour d'Arbois de Jubainville). D'Arbois de Jubainville vient discuter les hypothèses phonétiques des celtisants allemands tandis que Vendryes entreprend une description comparce des produits de l'évolution lexicale dans les différents dialectes;

—le français: 8 interventions (3 pour Joret, 2 pour Guerlin de Guer, 2 pour Bréal). Bréal traite d'étymologie; Joret et Guerlin de Guer présentent des travaux de dialectologie normande difficiles à exposer devant les cercles romanistes en raison, notamment, de la 
personnalité de J. Gilliéron et des réticences de G. Paris et P. Meyer pour une approche géographique qui délimite sur le

terrain l'aire des parlers;

—les langues indo-iraniennes: 7 interventions ( 3 pour Halévy, 2 pour Meillet). Halévy continue à trouver des rapprochements, très discutés, entre langues sémitiques et langues indo-iraniennes. Meillet cherche à étendre vers l'est le domaine comparatiste, non seulement en recourant aux enseignements du groupe indo-iranien mais en intogrant l'arménien et les langues slaves;

-le latin: 6 interventions ( 2 pour Bréal, 2 pour Lejay). Bréal prononce des communications sur les langes italiques, Lejay sur des problèmes de traduction de la littérature classique;

-le grec: 6 interventions (dont une étude d'Hubert Pernot sur

le grec moderne, 3 pour Bréal).

Par ailleurs, la mort de Rochemonteix et le retrait de Berger entraînent la disparition des interventions sur les langues couchitiques.

\section{0-1911: la découverte du monde}

Pour 47 communications, on relève un effacement de la plupart des domaines traditionnels (français, latin, langues romanes, langues celtiques et langues sémitiques oscillent entre une et trois communications) à une exception près, le grec, avec cinq interven tions pour cinq producteurs (dont Meillet)9. Dans le même temps, de nouveaux champs d'investigation s'ouvrent à la recherche avec le turc (J. Deny) et les langues caucasiques et, plus encore:

-les langues de l'Afrique Noire: 7 communications ( 3 communications de L. Homburger, 3 communications de M. Delafosse). Mais surtout le centre de gravité se situe désormais dans l'étude des langues indo-iraniennes: 14 communications (6 communica tions d'A. Meillet, 6 communications de R. Gauthiot). Elles sont devenues le secteur de référence de la S.L.P., où se marque sa précellence en matière de linguistique, celle aussi qui distingue les nouveaux dirigeants d'une génération précédente trop fidèle à l'ancien français (Paris), au latin (Havet) et au grec (Bréal). 
2.3. L'anabase de la S.L.P.

L'évolution de la S.L.P (tableaux 8 et 9) durant ses quarante 
premières années ressemble à un déplacement régressif dans l'espace et dans le temps. Les linguistes semblent remonter progressivement vers les sources supposées de la grande migration des langues indo-européennes (qu'on imagine alors en terme de déplacements de peuples). En 1872, on notait 15 communications sur le français, 15 sur le latin, 7 sur le sancrit (ou l'indo-européen), 4 pour le grec et 5 pour les langues sémitiques. Comme le montrent les tableaux 8 et 9 , et en intégrant les chiffres de 1872, on pourrait opérer un classement relatif qui donnerait la première place en 1872 au français (ex aequo avec le latin), en 1880 au latin, en 1890 au grec (ce que dissimule encore l'interventionnisme actif de Bréal) et, progressivement, à partir de 1900 aux langues indo-iraniennes. 
De 1900 à 1910, les explorations en Asie centrale, celles qui font des terres comprises entre les Indes, la Russie et la Chine, le domaine par excellence du Grand Jeu (Rudyard Kipling), concernent aussi les comparatistes dans la poursuite d'attestations plus archaïques. La $\sim$ découverte du sogdien et du tokharien relance les travaux de déchif- i frement dans l'espérance d'un rapprochement avec les formes . reconstruites de l'indo-européen. Les comparatistes privilégient désormais dans leurs discussions non seulement des méthodes qui = supposent un apprentissage particulier, sans comparaison avec ce qu'enseignent les Iycées, mais aussi des langues accessibles aux $\sim$ seuls spécialistes de l'indo-européen. L'exigence de professionnalisa- i tion assure la réputation de la S.L.P. mais tarit son recrutement.

Parallèlement, à l'intérieur d'un même domaine, d'autres déplacements se produisent. Les langues celtiques, absentes en 1890 et 1910, apparaissent en 1880 et 1900 dans la tension entre une 
dialectologie où la question du breton domine et une confrontation théorique entre les écoles française et allemande. En 1872, le sanscritisme était orienté vers la mythologie comparée, un thème abandonné dans les années qui suivent. Les communications consacrées au français traitent:

—en 1872, de 1'application de la grammaire historique aux problèmes du lexique (G. Paris, Meunier),

en 1880, de la recension des difficultés posées par 1'étymologie, dans une direction illustrée ultérieurement par A Thomas comme relevant de la «philologie française» (Godefroy, Joret),

—en 1890 de la question de 1'origine de 1'argot (Schwob),

—en 1900 et 1910, de la défense d'une conception dialectologique qui ne soit pas celle de la " géographie linguistique » de Gilliéron (Joret, Guerlin de Guer, M. Roques, O. Bloch).

De fait, le domaine de la S.L.P. s'est peu à peu rétracté:

— 1\$63-1X74: c'est la recherche d'une identité au fur et à mesure que sont écartés de la direction,

* les américanistes avec de Charencey (1863-1866),

* les hellénistes de l'enseignement secondaire conduits par Egger (1866-1871),

* les tenants de la mythologie comparce avec Gaidoz (18711873),

* les sémitisants (1873) (opposition d'Oppert et Halévy);

1874-1890: le primat des latinistes.

La recherche comparatiste privilogie le latin et s'isole à l'intérieur d'un champ linguistique français qui rompt sur des questions de méthode avec les Junggrammatiker allemands, sur des querelles de personnes avec l'école anglaise et, à propos des dialectes, avec l'école italienne. Les romanistes constituent un domaine réservé autour de la Revue des Langues Romanes (1870) et de Romania (1872), abandonnant à la S.L.P. les marges de leur domaine: la dialectologie et les argots. La mythologie comparée, déconsidérée par des hypothèses mal fondées, et le sémitisme, déchiré par des querelles d'école, sont réduits à la portion congrue.

—1890-1905: la domination précaire des hellénistes.

Déjà le grec et plus encore le latin sont en recul, suite au retrait de Havet qui se consacre de manière croissante à d'autres pério

_S $\sim \mathrm{q} \sim \mathrm{ys} \sim 111 \mathrm{R}-\sim 11 \ll .11$ - - N1IB1 - Yffle $\sim \mathrm{al} \sim 1 \sim$ - Nleata. 11 
diques'• et de Bréal, très affecté par la mort de sa femme. En dépit de quelques interventions de latinistes venus de l'Institut Catholique de Paris, tel l'abbé Lejay, les humanités restent l'affaire de producteurs scolaires pour le moins réservés à l'égard des méthodes en vigueur à la S.L.P. Le grec a pour représentants attitrés Alfred et Maurice Croiset, le latin Gaston Boissier; les langues classiques, étudiées surtout au lycce et dans les facultés de lettres, ne participent pas au renouvellement de l'analyse comparatiste ou historique.

—à partir de 1900, le progrès des langues indo-iraniennes.

Elles s'imposent comme les représentantes les plus légitimes d'une linguistique qui se sépare de l'anthropologie. Alors qu'Eugène Burnouf ( $\mathrm{t}$ 1852) et Abel Bergaigne ( $\mathrm{t}$ 1888) se donnaient pour fin la compréhension des religions indiennes, Gauthiot ( $\mathrm{t}$ 1916) privilégie une approche épigraphique et linguistique. La Société Asiatique, qui continue de rassembler toutes les disciplines de l'orientalisme, se sépare de la S.L.P. qui se focalise sur l'analyse du système linguistique de langues peu accessibles, plus souvent mortes que vives, comprises entre l'Inde et la Grèce. Dans l'entre-deux-guerres, une part de l'investigation des indoeuropéanistes français, notamment Benveniste et Dumézil, s'inscrit dans la continuité d'un travail de description phonologique et grammatical privilégiant une zone comprise entre le Caucase et l'Afghanistan.

\subsection{La S.L.P. d'A. Meillet}

Les débuts de la S.L.P., jusqu'à 1880, ont marqué le passage du témoin d'Egger à Bréal qui, après que Saussure eut renoncé à lui succéder, dans les premières années du xxe siècle, confie à son tour les destinces de l'association à Antoine Meillet (1866-1936). Auditeur à 1'É.N.S., inscrit comme élève titulaire à la IVe section de 1'É.P.H.É. en juillet 1887, cacique à l'agrégation de grammaire en 1889, Meillet est nommé le 20 novembre de la même annce répétiteur de grammaire comparée à 1'É.P.H.É., un poste précaire qui lui évite de rejoindre son affectation dans un lycée.

Après la démission de Saussure en 1891, 1'assemblée de la 
IVe section ayant attribué par seize voix (contre treize pour Meillet) cet enseignement à Louis Duvau, G. Paris obtient que lui soient rétrocédés les émoluments de la conférence d'hébreu rabbinique supprimoe cette annce-là. Au procès-verbal entérinant la décision, Gaston Paris joint une lettre non officielle, expédiée de son château de Cerisy, dans laquelle il précise au directeur de l'enseignement supérieur:

« Ce jeune homme est, au dire de Saussure, de Bréul, de Darmesteter qui le suivent depuis trois ans, un esprit tout à fait original et fécond, peut-être un génie; il aurait été tout à fait dommage de ne pas lui ouvrir la seule voie qui lui convienne et où il puisse rendre à la science tous les services qu'elle est en droit d'attendre de lui»t'.

En 1894, après la disparition de James Darmesteter, Meillet est promu directeur-adjoint pour l'enseignement du zond. I1 soutient sa thèse en 1897: Recherches sur l'emploi du génitif-accusatif en vieux-slave et obtient la chaire d'arménien aux Langues Orientales quand Carrière l'abandonne en 1902 avant de succéder à Bréal au Collège de France en 1906. À quarante ans, Meillet cumule donc des charges d'enseignement dans les trois institutions d'enseignement supérieur où l'étude des langues est représentée, en dehors de la faculté des lettres de Paris.

Présenté par Bréal et Havet à la S.L.P. en 1889, il y multiplie les interventions et les articles et en devient le secrétaire tout désigné lorsque Bréal renonce à occuper le poste. I1 joue un rôle décisif dans une orientation de la recherche qui abandonne le latin et le grec aux maîtres du secondaire et réserve aux comparatistes le groupe indo-iranien et la découverte de nouvelles aires linguistiques distribuées à ses proches et ses élèves dans un souci d'exhaustivité qui s'accomplit avec la rédaction des Langues du monde (1924, en collaboration avec Marcel Cohen).

Ses comptes rendus dans le Bulletin lui assurent une position d'arbitre: il en rédige les deux tiers de 1910 à 1912, les trois quarts en 1913, les neuf dixièmes en 1914, parfois tous pendant la durce de la guerre. Pour la première fois, en 1910, présentant le recueil d'articles publié en hommage à Louis Havet t2, Meillet commente sa propre contribution, lui consacrant une part double 

ou triple de celle qu'il accorde aux autres à l'exception de celle de Psichari qu'il fustige avec une exceptionnelle sévérité. I1 récidive en 1913 pour sa grammaire de l'arménien, parue en Allemagne, et désormais se rend à lui-même le service de dire le bien qu'il pense de son œuvre. La guerre, en éclaircissant les rangs, lui laisse une position de maîtrise absolue: comme le note Mounin, il ffit le gardien vigilant de la grammaire historique au prix d'un autoritarisme qui le conduisit à sous-estimer la valeur de certains de ses élèves (Dumézil s'en plaint) et d'un dogmatisme qui lui firent méconnaître les apports de la phonologie ou de certains grammairiens hétérodoxes.

En même temps que, de 1880 à 1910, la S.L.P. s'est constituce en référence obligée de la linguistique en France, la coupure avec le champ international de la linguistique, que la défaite allemande accusera encore, est devenue irréversible. Au moment où Meillet accède au secrétariat, le comparatisme ne parvient plus à s'étendre dans l'enseignement supérieur, réduit aux postes acquis dès avant 1885 à l'É.P.H.É., au Collège de France, aux Langues Orientales, à l'École des Chartes, un ensemble d'institutions à effectif limité qui ne pouvaient assurer de débouchés à la nouvelle génération, ce dont Meillet lui-même faillit pâtir.

Face à la résistance de facultés de lettres vouces à privilogier I'enseignement et les concours de recrutement, la S.L.P. figure une instance de recherche prestigieuse et respectée mais sans concurrence et presque sans public. Antoine Meillet paraît dominer une scène internationale où l'Allemagne a reculé sans que soit pris en compte l'apport de nouvelles écoles (tchèque, danoise, soviétique, américaine), en marge des pays traditionnellement dominants. Du moins le cercle actif de la S.L.P. aura imposé qu'il y ait, pour toute question intéressant les sciences du langage en France, un lieu de confrontation et un jugement interne, propre aux pairs, en sorte que l'appréciation du travail ne soit pas abandonnce à des instances qui auraient numériquement dominé les comparatistes (I'enseignement secondaire ou les universités). Bien que les linguistes aient dû renoncer à s'imposer dans les filières éducatives classiques t3 et dans les concours de recrutement, se 
condamnant par là même à une certaine marginalité, ils ont du moins préservé l'indépendance de leurs formations par rapport à

ceux-ci et la continuité d'un travail voué à l'analyse exclusive des

langues.

29, rue Basse d'Ingré

Gabriel BERGOUNIOUX,

45000 Orléans

Université d'Orléans

BIBBIOGRAPHIE

AUROUX, Sylvain (éd), 1989: «Antoine Meillet et la linguistique de son temps», Histoire Epistémologie Langage, 10/2

BERGOUN[OUX, Gabriel, 1991: «L'introduction de l'ancien français dans l'université française (1870-1900)», Romania 445-446, 243-258

—, 1994: Aux origines de la linguistique franS:aise, Paris, Agora-Pocket

— , 1996: «Aux origines de la Société de Linguistique de Paris», BSL 91/1, 1-36

Bulletin de la Société de Linguistique de Paris, volume 6 (1885) à 19 (1915)

CHARLE, Christophe; FERRÉ Régine, 1985: Le personnel de l'enseignement supérieur en France aux $\mathrm{xlxe}$ et $\mathrm{xx} \sim$ siècles, Pans, Éditions du CNRS.

CHARLE, Christophe, 1987: Les élites de la République 1880-1900, Paris, Fayard

CHEVALIER, Jean-Claude; DELESALLE, Simone, 1986: La linguistique, la grammaire et l'école 1750-1914, Armand Colin

DÉClMO, Marc, 1995: «Victor Henry (1850-1907) ou 1'itinéraire d'un linguiste autodidacte d'après les fragments de sa correspondance ", Archives et documents de la SHESL, seconde série, n• 12 
ESPAGNE, Michel, WERNER, Michael, 1990: Philologiques I: contribution à I histoire des disciplines littéraires en France et en Allemagne au xlxe siècle, Paris, Éditions de la Maison des Sciences de l'Homme MEILLET, Antoine, 1975: Linguistique historique et linguistique générale, Paris, Honoré Champion PROST, Antoine, 1968: Histoire de l'enseignement en France 1800-1967, Paris, Armand Colin VENDRYES, Joseph, 1923: Le langage. Introduction linguistique à l'histoire, Paris, La Renaissance du Livre , 1955: La Société de Linguistique de Paris (1865-1955), Ortis 4, 7-21 
Having found an equilibrium after 1876 under the direction of young comparative Indo-Europeanists, the Société de Linguistique de Paris became the reference point for language science, welcoming research which was scarcely represented in faculties of letters: phonetics, dialectology and slang studies, and the Celtic and Cushitic or «Sudanese» languages. The analysis of the papers and contributions which appeared in the Société's Mémoires allows us to pinpoint the dominant themes of discussion around 1870 (the historical grammar of French), 1880 (Latin metrics and etymology), 1890 (Grcek dialectology and etymology) and the years preceding the first world war (Indo-Iranian comparativism and decipherment). This « anabasis » in time and space (from Old French to the ancient representatives of Indo-Iranian) was accomplished under the influence of the first two secretaries: Bréal (1832-1915) and Meillet (1866-1936).

\section{LA SOCIETÉ DE LINGUISTIQUE DE PARIS (1876-1914)}

A1 encontrar su equilibrio a partir de 1876 y bajo la direccion de unos jovenes comparatistas especialistas en lenguas indoeuropeas, la Société de Linguistique de Paris se convierte en el centro de referencia de las ciencias del lenguaje, amparando investigaciones poco representadas en las facultades de letras: fonética, dialectologia y argots, lenguas célticas, cusiticas o « sudanesas ». El analisis de las comunicaciones y contribuciones aparecidas en las Mémoires de la Sociedad permite situar el tema dominante de los debates hacia 1870 (gramatica historica del francés), 1880 (métrica y etimologia latinas), 1890 (dialectologia y etimologia griegas) y hacia los anos anteriores a la primera guerra mundial (comparatismo indoiranio y desciframiento). Semejante «anabasis» temporal a la vez que espacial (desde el francés antiguo hasta los representantes arcaicos del grupo indoiranio) se cumple bajo la influencia de los dos primeros secretarios: Bréal (1832-1915) y Meillet (1866-1936). 